\title{
Position Based Density Conscious Routing Protocol in Vehicular Ad Hoc Networks
}

\author{
Anant Ram and Manas Kumar Mishra \\ GLA University, Mathura, India \\ anant.ram@gla.ac.in,manas.mishra@gla.ac.in
}

\begin{abstract}
Vehicular ad hoc networks (VANETs) have high mobility of vehicles resulting in frequent disconnections in routes. Geographic routing protocols are commonly preferred in VANETs as they do not require, route formation prior to forward the data packets as well as route maintenance. Most of the position based routing protocols adopt the greedy mode to establish the route and switch to some recovery mechanism like perimeter routing, in case of failure. Due to high mobility characteristic, selection of next forwarder based on greedy approach basically affects the performance of the routing protocols. At the same time neighborhood density of a vehicle play significant role for the selection of next forwarder toward to the direction of destination. In this paper, we propose a routing strategy that uses the restricted greedy forwarding to select next best fit forwarder, which will reduce the frequency of switching to recovery mode. Vehicle seeking the next forwarder will consider neighborhood vehicles having a sufficiently dense neighborhood and the vehicle out of these considered set having the least standard deviation of average relative velocity with its own neighboring vehicles will be selected as the next forwarder. The objective is to increase the longevity of the route and thus increasing the throughput without greatly affecting the end to end delay. The performance of the proposed approach is compared with E-GyTAR and GPSR, and the simulation results are presented for both highways as well as city scenarios.
\end{abstract}

Keywords: Best fit forwarder; VANETs; Restricted Greedy Forwarder

\section{Introduction}

Vehicular Ad hoc Networks (VANETs) play key role in realizing intelligent transportation systems. These networks also have tremendous potential in applications targeting road safety, acquisition of current traffic and weather information, sharing of multimedia information etc. The communication over VANETs can be broadly classified into two different categories like Vehicle to Vehicle communication (V2V), Vehicle to infrastructure (V2I). In V2V communications, vehicles transfer information among themselves, without the help of road side units. However, in the V2I communications, the road side units also become a part of, and actively participate in the VANETs. In case of $\mathrm{V} 2 \mathrm{~V}$ communication every vehicle is considered to have been installed with various onboard sensing units, which allow large scale sensing, decision making and controlling actions to perform a number of tasks that arises in wireless communication system. The wireless communication system in VANETs adopts certain technology from IEEE802.11 with some modification like IEEE 802.11p. United States, Federal Communications Commission (FCC) has allotted $75 \mathrm{MHz}$ of licensed spectrum from $5.85 \mathrm{GHz}$ to $5.925 \mathrm{GHz}$ for Intelligent Transport Systems (ITS). The allocated band support seven channels each of $10 \mathrm{MHz}$. Moreover, the wireless communication system for VANETs (V2V) can provide a broadcast range between 200 to 1000 meters [8]. Most of the

Received (March 11, 2017), Review Result (September 5, 2017), Accepted (September 7, 2017) 
research studies consider that, due to the high mobility factor associated with V2V communications, the packets are either get dropped due to connectivity issues, or the end to end delay is increased. Subsequently, lot of work in literature adopts greedy forwarding strategy for their routing [7].

Further, in case where end to end path may not be viable based on this strategy, these works defer the strategy and establish route based on the perimeter routing [5], instead. However, the shifting back to the greedy strategy will largely depend on time and mobility to make the neighborhood of the current forwarder conducive enough for such a shift. Failing which the current forwarder vehicle is supposed to carry packets until a next forwarder is available which is nearer to the destination. The delay caused by this temporary hold in the forwarding of the packets may negatively affect the end to end delay. The density of vehicles will be very high in daytime whereas at night time it may very less. Further, the density on the road will vary based on occasion like festivals, weather and other social and environmental factors. Moreover, the velocity of the vehicles moving in a particular direction is highly dependent on the traffic density and is likely to inherit the variation of density as well. Due to this density variation and limitation of available bandwidth, the information propagation may suffer [4]. This paper targets to make the position based routing protocol adaptive to such variations in density and velocity in the network. The paper is organized as follows; Section 2 describes the related work and motivation behind the work. Section 3 presents the proposed methodology. Section 4 presents the proposed density based routing protocol. In Section 5, we present the experimental evaluation of the proposed work. Finally Section 6 concludes the paper. Appendix gives the mathematical analysis of link and route life time along with the link reliability.

\section{Related Work}

VANET is the sub class of Mobile ad hoc network. A number of efficient routing algorithms have been proposed to send the packets from source to destination in MANETs, like AODV [16], DSR [6], TORA [15] etc. These routing algorithms do not perform well in VANETs as compared to MANETs due to high mobility of the vehicles. Therefore, routing protocols for VANETs need to be dealt separately, and are to be designed with mobility being the core objective.

Most of the routing approaches in vehicular ad hoc networks combine both ways of communication i.e., V2V and V2I. to get the desired results. Vehicular networks are highly dynamic in nature and this affecting the routing and packet delivery ratio. In addition, performance of vehicular routing protocols is also susceptible to the vehicular density. The most efficient routing protocols are real time connectivity aware protocols. These protocols are having the ability to reactively discover connected paths to the destination by flooding the networks with a route request message and computing dynamically the density of the vehicles. still flooding introduces the bandwidth wastage. It is just because that these routing strategies do not consider the location services. Once these strategies select the path from source to destination, start the message transfer. The selected path may disconnect over the time CAR [13], in it the source vehicle broadcasts probe messages to the destination. The destination vehicle decides the path for further communication and sends back to the source vehicle. The source vehicle follows the same path followed by the packets forwarded by the destination. In case the route is to be updated, then the request message carries the change of direction information along with hop count information for a route. This protocol may introduce more control overhead as a consequence of starting a new path discovery. One remedy ACAR [18] and AGP [17] suggested by these, is simply to keep and carry the packet until there exist available next hop. This recovery strategy work fine if the disconnection is for short duration. In Social grouping based routing protocol SGBR [1], the authors focus on increasing the throughput 
by spreading the multiple copies of same packet over the network with the probability that at least one copy of the packet will be reached to the destination. It also estimates the available buffer space and the occupied buffer space. However, the drawback is the overuse of the bandwidth for any communication i.e., misuse of the bandwidth due to redundant copies of the same packet available over the network. Density Aware Emergency Message Extension Protocol DEEP [3] focuses on delay and reliability of emergency messages while achieving a trade-off between the two conflicting parameters. The protocol guarantees the delivery of emergency information in timely manner. While the Greedy Bundle Relaying Scheme with Bulk Bundle Release (GBRS-BBR) [10] allows source to greedily release a bulk of bundles to every arriving vehicle irrespective of its arrival time and speed, which are predicted to contribute the most minimization of overall bundle delivery delay. On the other hand the Delay-Aware Data Delivery in Vehicular Intermittently Connected Networks (DADD) [9] allows a source stationary roadside unit to carry out necessary bundle retransmission to high speed vehicles newly entering in its communication range and these vehicles guarantee the minimum delay data delivery to the destination stationary roadside unit.

In this paper we purely focus on V2V communication based routing protocols. The use of digital maps, GPS receivers and navigation system in modern vehicle inspired the study of position based routing in vehicular networks. The position-based routing protocol GPSR [7] depends on the location service. In this approach, the sender requires the position information of itself and the destination to initiate the message transfer. It uses two strategies to forward the packet from source to destination based on position information, one is greedy forwarding and the other one is perimeter routing. In greedy forwarding, the sender selects a neighbor as the forwarding vehicle if it has the shortest Euclidian distance to the destination among all neighbors. On the other hand, if it encounters a void region, then perimeter routing is used to select the next forwarder. The recovery strategy is not efficient for high dynamic nature of vehicular ad hoc networks. It is suitable for highways scenarios while the performance of the same is poor for city environment.

Geographic source routing GSR [11] is specially designed for routing in city scenarios to overcome the limitations of GPSR. The perimeter forwarding specially restrict the advantage of greedy forwarding in city scenarios. GSR uses location information of the vehicle and the street map. The sender vehicle calculates trajectory by using Dijkstras shortest path algorithm from sender to the destination and also computes a sequence of intersections. The sequence of intersections is placed in the data packet header when source forwards the message to the destination vehicle. While the drawback of GSR is that it does not consider vehicular traffic on street due to this the shortest path is not the optimal one.

A-STAR [2] is used in city scenarios. It eliminates one of the drawbacks of GSR by considering the vehicular traffic on the street. A-STAR targets urban area traffic and uses the city bus route information to identify anchor point. The purpose of this proposal is to deliver more packets by using the same road segment, which is having more connectivity. It introduces new recovery strategy for packets get stuck in local maximum problem, by avoiding the packet forwarding using those street segments, where local maximum occurred is marked. The performance of A-STAR is better than GSR and GPSR. Moreover most of the traffic is shifted towards the major streets, which causes bandwidth congestion.

GPCR [12] enhances the work proposed GPSR [7] and applies restricted greedy forwarding of packets. It selects junction vehicles as next forwarders. The selected junction vehicle is known as the coordinator and the coordinator vehicle is not necessarily the closest vehicle to the destination. The recovery strategy in GPCR remains the same as GPSR. The performance of GPCR is better than the GPSR. However there is the possibility that packets loop back in the same street from which the packet has arrived. 
An improvement to geographical source routing named GyTAR[5] is an intersection based geographical routing protocol that calculates a sequence of intersections between source and destination. It considers the parameters like remaining distance to the destination and the traffic density variation in VANETs. It follows either an improved greedy forwarding mechanism or a carry-and-forward mechanism to forward the message from source to destination vehicle, depending upon the availability of the vehicles to behave as the forwarding vehicle. The source vehicle finds the position of destination vehicle by using grid location services. Digital maps are used to identify the position of junctions and also to find the shortest path towards destination by using Dijkstra shortest path algorithm. The performance of GyTAR is better than GSR. However it also has some limitations, like junction selection mechanism does not consider direction of the vehicle. Enhanced GyTAR (E-GyTAR) [19] is the modification of of GyTAR routing protocol which is designed for city environment. In E-GyTAR, Junction selection mechanism is based on vehicle traffic density in the direction of the destination. It remove the limitation of GyTAR by considering the direction of the vehicles before the selecting the next junctions. Thus, selected junction has the higher traffic density in the direction of the destination. Still there are some issues in E-GyTAR that it misses' non directional paths which may provide the shortest path having enough connectivity to route the packets towards the destination.

In this article we proposed an approach i.e. Position Based Density Conscious Routing (PBDCR) Protocol, which take the base of location services to have the information of the destination vehicle. Most of the above mentioned position based routing strategies use variety of matrices for selection of next forwarding vehicle like relative velocity, maximum distance toward to the destination which is nearer to the destination, less angle variation toward to the destination etc. But our proposed approach mainly focuses on neighborhood density and standard deviation of average relative velocity, for the selection of next forwarder. Each vehicle is equipped with on-board navigation system which determines the position of neighboring junctions in city environment and also provides useful street level information through the use of pre-loaded digital map. The performance of PBDCR protocolis better with respect to E-GyTAR and GPSR in both city as well as highway as scenarios.

\section{Proposed Methodology}

Position based routing protocols works in two modes i.e., greedy mode and recovery mode. In greedy mode every packet holder selects the next forwarder, in its communication range, which is nearer to the destination for some position based routing protocols and for some it may not nearer to the destination. Further, route life from source to destination is affected by high speed variation and density variation of the vehicles in VANETs. The high speed variation may result in frequent route disconnection, will result as increase in the probability of switching from greedy mode to recovery mode for most of the position based routing protocols. Vehicle speed variation causes frequent link changes, and may result in erroneous transmissions. This can make the protocol very inefficient particularly in environment like VANETs, where radio bandwidth is a scarce resource. Therefore, there is a need for efficient and robust routing scheme for VANETs.

The proposed approach selects the next hop forwarder toward to the destination which may or may not be nearer to the destination. The message holder vehicle observes the characteristics of its surrounding vehicles under its communication range, like density of the vehicles, standard deviation of average relative velocity of the vehicle. We propose a position based density conscious routing protocol. Like most of the position based routing strategies, we also assume that the sender vehicle is aware of the position of the destination by virtue of some location service like Grid Location Service (GLS). Further, we assume that each vehicle is equipped with on board sensing units and Global 
positioning systems. The proposed work considers highway as well as city as the scenario where vehicles are moving with varying speed. Every pair of vehicles that are positioned under communication range of each other will be considered to be connected. The V2V interactions are modeled by using the following entities.

Sender: The vehicle that initiates the message that is to be transmitted to some identified destination vehicle.

Receiver: The vehicle that is supposed to receive the message that is transmitted from the sender vehicle.

Next Best Fit Forwarder: The vehicle which is selected from the neighborhood of the current forwarder and is the best among the neighbors based on the selection criteria. This vehicle is supposed to forward the message to its next best fit forwarder till the message reaches the destination vehicle. The sender behaves as the current forwarder for the first hop best fit forwarder selection. Table 1 presents the symbols used to represent various system parameters.

Table 1. Symbols Used to Represent System Parameters

\begin{tabular}{|c|c|}
\hline Symbol & Description \\
\hline $\mathrm{N}_{\mathrm{R}}(\mathrm{A})$ & Set of vehicles in the neighborhood of vehicle A \\
\hline $\mathrm{V}_{\mathrm{var}}(\mathrm{A})$ & $\begin{array}{l}\text { Average relative velocity variance of vehicle } A \text { with its neighbor vehicles } \in \\
N_{R}(A)\end{array}$ \\
\hline$\lambda$ & $\begin{array}{l}\text { Threshold value of neighborhood density for a vehicle } A \text { to initiate the next } \\
\text { best fit forwarder selection process (i.e. } \mathrm{FN}_{\mathrm{R}}(\mathrm{A}) \geq \lambda \text { ) }\end{array}$ \\
\hline$\delta$ & $\begin{array}{l}\text { Threshold value of standard deviation of average relative velocity for a } \\
\text { vehicle } A \text { to contend as the next best fit forwarder (i.e. Standard Deviation of } \\
\text { average relative velocity for vehicle } A \leq \delta \text { ) }\end{array}$ \\
\hline $\mathrm{BFF}_{\mathrm{A}}$ & Best Fit Forwarder vehicle for the current forwarder vehicle A, (say B) \\
\hline LLT $_{\mathrm{AB}}$ & Contact Life Time of vehicle A with its Best Fit Forwarder vehicle B \\
\hline RLT & $\begin{array}{l}\text { Route Life Time i.e. } \min \left(\text { all } \mathrm{LLT}_{\mathrm{AB}} \text { on the entire route from source to }\right. \\
\text { destination) }\end{array}$ \\
\hline $\mathrm{R}$ & Transmission Range for each vehicle \\
\hline
\end{tabular}

\section{Lemma 1: Adaptive Neighborhood Density}

The density of all the density connected vehicles can be considered as a random variable and its expected value can be determined. Let us assume that, each vehicle has sufficient density in its neighborhood and hence, the density can be considered as contiguous event. Thus, the expected density in the neighborhood of any vehicle can be modeled as follows: Suppose, a vehicle A has $\mathrm{n}$ neighbors in the direction of destination vehicle and let $\lambda_{1}, \lambda_{2}, \ldots, \lambda_{\mathrm{n}}$ be the neighborhood density of all its $\mathrm{n}$ neighbor vehicles, respectively. Because all $\mathrm{n}$ neighbor vehicles are under communication range of vehicle A, So vehicle A receives the density information in HELLO beacon message periodically. Based on the above received information by every vehicle calculate the expected value of $\lambda$ with respect to its neighbors in the direction of destination as follows:

$$
\begin{aligned}
& \lambda_{\max }=\operatorname{Max}\left(\lambda_{1}, \lambda_{2}, \ldots \ldots \ldots \ldots, \lambda_{\mathrm{n}}\right) \\
& \lambda_{\min }=\operatorname{Min}\left(\lambda_{1}, \lambda_{2}, \ldots \ldots \ldots \ldots, \lambda_{\mathrm{n}}\right)
\end{aligned}
$$

Let $F(\lambda)$ and $f(\lambda)$ be the Cumulative Distribution Function(CDF) and Probability Density Function(PDF) of $\lambda$, respectively. Then the Cumulative Distribution Function 


$$
\begin{aligned}
\mathrm{F}(\lambda) & =\mathrm{P}\left\{\left\{\lambda_{1} \geq \lambda, \lambda_{2} \geq \lambda_{3}, \ldots \ldots \ldots \ldots, \lambda_{\mathrm{n}} \geq \lambda\right\}\right. \\
& =\prod_{\mathrm{i}=1}^{\mathrm{n}} \mathrm{P}\left[\lambda_{\mathrm{i}} \geq \lambda\right]=\left(\frac{\lambda}{\lambda_{\max }}\right)^{\mathrm{n}}
\end{aligned}
$$

And the probability density function

$$
\mathrm{f}(\lambda)=\frac{\mathrm{d}}{\mathrm{d} \lambda} \mathrm{F}(\lambda)=\frac{\mathrm{n}}{\lambda_{\max }}\left(\frac{\lambda}{\lambda_{\max }}\right)^{\mathrm{n}-1}
$$

Then the expected value of $\lambda$ is will be as follows:

$$
\begin{gathered}
\mathrm{E}(\lambda)=\int_{\lambda_{\min }}^{\lambda_{\max }} \lambda \mathrm{f}(\lambda) \mathrm{d} \lambda \\
\mathrm{E}(\lambda)=\int_{\lambda_{\min }}^{\lambda_{\max }} \lambda \frac{\mathrm{n}}{\lambda_{\max }}\left(\frac{\lambda}{\lambda_{\max }}\right)^{\mathrm{n}-1} \mathrm{~d} \lambda \\
\mathrm{E}(\lambda)=\frac{\mathrm{n}}{\lambda_{\max }}\left(\frac{\lambda_{\max }{ }^{\mathrm{n}+1}-\lambda_{\min }{ }^{\mathrm{n}+1}}{\mathrm{n}+1}\right)
\end{gathered}
$$

Thus the value of $\lambda$ for the vehicle $A$ will be equal to the value of $E(\lambda)$.This way every vehicle calculate the neighborhood density threshold value $\lambda$ for the selection of next best fit forwarder.

\section{Lemma 2: Direct Density Connected Vehicle}

Two vehicles are said to be direct density connected if they are under the communication range of each other. Suppose, vehicle A is direct density connected to the vehicle $B$. Then, the neighborhood of a vehicle $A$ will be,

$$
\mathrm{N}_{\mathrm{R}}(\mathrm{A})=\left\{\mathrm{B} \mid \forall \mathrm{B} \in \mathrm{N}_{\mathrm{R}}(\mathrm{A}) \text { and Euclidian distance }(\mathrm{A}, \mathrm{B}) \leq \mathrm{R}\right\}
$$

\section{Lemma 3: Indirect Density Connected Vehicles}

Two vehicles are said to be indirect density connected vehicles if they are having a sequence of direct density connected vehicles in between them. The vehicles $\mathrm{A}$ and $\mathrm{C}$ are said to be indirect density connected vehicles because there is one vehicle B which is directly density connected with both vehicle A and C. Based on transitive property, if there is a sequence of density connected vehicles then they are said to be indirect density connected vehicles apart from the direct density connected vehicles.

\section{Lemma 4: Forward Density Connected Vehicle}

A vehicle A is said to be Forward Density Connected FDC (A) if it satisfies the following condition

$$
\left|\mathrm{FN}_{\mathrm{R}}(\mathrm{A})\right| \geq \lambda \text {. }
$$

Where, $\mathrm{FN}_{\mathrm{R}}(\mathrm{A})=\left\{\mathrm{B} \mid \forall \mathrm{B} \in \mathrm{N}_{\mathrm{R}}(\mathrm{A})\right.$ where $\mathrm{X}_{\mathrm{A}} \leq \mathrm{X}_{\mathrm{B}}$ and Euclidian distance(A, $\left.\mathrm{B}\right) \leq \mathrm{R}$ \} and $\left(X_{A}, Y_{A}\right)$ and $\left(X_{B}, Y_{B}\right)$ are the position information of vehicle $A$ and $B$ respectively.

Lemma 5: Average Relative Velocity of Vehicle A: if $v_{A}$ be velocity of vehicle A and $v_{B}$ be the velocity of every vehicle $B \in N_{R}(A)$ then the average relative velocity of vehicle A with respect to the neighborhood will be as follows 


$$
\mu_{\mathrm{A}}=\frac{\sum_{\forall \mathrm{B} \in \mathrm{N}_{\mathrm{R}}(\mathrm{A})}\left|\mathrm{v}_{\mathrm{A}}-\mathrm{v}_{\mathrm{B}}\right|}{\left|\mathrm{N}_{\mathrm{R}}(\mathrm{A})\right|}
$$

Lemma 6: Average Relative Velocity Variance: - Average relative velocity variance of a vehicle A with its surrounding vehicles is defined as follows:

$$
\mathrm{V}_{\mathrm{var}}(\mathrm{A})=\frac{\sum_{\forall \mathrm{B} \in \mathrm{N}_{\mathrm{R}}(\mathrm{A})}\left(\mu_{\mathrm{A}}-\mu_{\mathrm{B}}\right)^{2}}{\left|\mathrm{~N}_{\mathrm{R}}(\mathrm{A})\right|}
$$

\section{Lemma 7: Best Fit Forwarder}

A vehicle $B\left(B \in N_{R}(A)\right)$ is said to be best fit forwarder for a vehicle $A$, if it satisfies the following conditions:-

1) $B \in \mathrm{FN}_{\mathrm{R}}(\mathrm{A})$

2) $\operatorname{FDC}(\mathrm{B})$

3) Standard deviation of average relative velocity for vehicle $\mathrm{B} \leq \delta$

4) $\left|v_{A}-v_{B}\right|$ should be less than a predefined Threshold.

If a vehicle $B$ satisfies all the above conditions for vehicle A, then the Best Fit Forwarder BFF (A) will be vehicle B.

\section{Proposed Algorithm (PBDCR)}

In this paper we propose a position Based Density Conscious Routing Protocol (PBDCR). Every vehicle sends the HELLO message to its neighboring vehicles, which carry the information such as position, velocity of itself, average relative velocity, neighborhood density, Forward Density Connected Status i.e., True or False and the standard deviation of average relative velocity of itself with respect to its neighborhood. Every vehicle initially sends the average relative velocity of itself as zero, standard deviation of average relative velocity of itself as zero, also Forward Density Connected status as false. After initial round onward, based on the received position and velocity information of its neighborhood vehicles in HELLO message, each vehicle calculates the average relative velocity and forward density connected status. In next round of HELLO beacon message, based on the received average relative velocity information from its neighboring vehicle, every vehicle calculates standard deviation of average relative velocity and also checks the status of Forward Density Connected, whether true or false. Every vehicle periodically exchanges the above information in HELLO message with its neighbors.

A source vehicle $S$ sends a packet to the destination D. First it obtains the position information of the destination vehicle by using the location services. Then the source currently having the packet to forward, then selects the next best fit forwarder by using the following algorithm. This way the selection of next forwarder increases the route life time and packet delivery ratio. If it fails to find the next vehicle as best fit forwarder based on selection criteria. Then it compromises with its selection strategy and handover the packet to the vehicle toward to the destination which is in its communication range and absolute velocity difference is less. Another case may arise that packet holder vehicle does not have the vehicle in the direction of the destination then it carry the packet for certain specified time duration as threshold and try to find out the next vehicle in its communication range. If it finds, then it handover the packet to that vehicle. The sensing of next vehicle in the recovery mode is based on the periodical HELLO beacon message. If the current packet holder vehicle which enters into recovery mode does not find the next vehicle in the specified duration then it send the error message to the source vehicle to stop the packet sending. The source vehicle again reforms the next best fit forwarder selection process and keeps sending the packets to the destination. 
In city Scenarios, The optimal path based on the above density notion will contain the set of sequence of anchor points (intersection) through which a packed will routed to reach the destination. Since we assume that each vehicle is equipped with GPS and digital map of the city. Based on the city map information, the source vehicle determines the set of intersections and the road segments with sufficient vehicle density toward to the destination. It delivers the packet to the anchor vehicle as a next best fit forwarder, if it satisfies the Best Fit Forwarder condition. Otherwise it carries the packet and search the next best fit forwarder based on the selection criteria. Since we adopt the density based notion for selection of next forwarder toward to the destination, which very well taken the consideration of road segments with sufficient density toward to the destination. This density notion based road segment selection ensures the reduced switching of routing from restricted greedy forwarding to the perimeter mode. The standard deviation of average relative velocity based next best fit forwarder selection ensures the increased route life time. The forward density connected status specifies that the selection of all the forwarder from source to the destination along with the anchors based on lemma4 guarantee about the link as well as route reliability. In city scenarios especially at junctions, each vehicle take care of calculation of parameters like standard deviation of average relative velocity and forward density connected status based on the movement direction of the vehicle. Which helps the selection of next best fit forwarder toward to the destination. The recovery strategy for the case of local maxima in the proposed approach is carry and forward.

\section{Algorithm for Selection of Best Fit Forwarder:}

Require: A network of vehicles with the current forwarder being the vehicle A

Ensure: Selection of Best Fit Forwarder say vehicle B for vehicle A

If Destination Vehicle is within the transmission range of the Source vehicle then Source will transmit the packet directly to the Destination

else

for each vehicle $B \in N_{R}(A)$ do

if $B F F(A)$ then

Enqueue $\left(\mathrm{V}_{\mathrm{B}}\right)$

else

Continue

end if

end for

ifQueue != NULL then

$\operatorname{BFF}(\mathrm{A})=\min \left(\left|\boldsymbol{v}_{\boldsymbol{A}^{-}} \boldsymbol{v}_{\boldsymbol{B}}\right|\right) ; / /$ Extract the minimum velocity difference vehicle from the queue

else if Queue $==$ NULL

$\mathrm{BFF}(\mathrm{A})=\min \left(\left|\boldsymbol{v}_{\boldsymbol{A}^{-}} \boldsymbol{v}_{\boldsymbol{B}}\right|\right) ; / /$ Extract the minimum velocity difference vehicle from the $\mathrm{N}_{\mathrm{R}}(\mathrm{A})$ toward to the destination

else if carrying time $<T_{w}$

Sense the next hop in the direction of destination and handover the packet

else send the error message to the source to stop the packet sending

end if end if

end if

end if 


\section{Simulations and Result Analysis}

The performance of the proposed approach PBDCR E-GyTARand GPSR has been compared by simulation using NS-2.34. The realistic vehicular mobility trace has been generated using SUMO (Simulation of Urban MObility) and MOVE. The vehicular traffic scenario consisting of roads, traffic lanes on road, junctions etc. has been generated using SUMO. The proposed approach selects the next best fit forwarder, among the potential forwarders of the current forwarding vehicle, based on the standard deviation of average relative velocity and the potential forwarders neighborhood density. The objective behind this approach is to positively influence the longevity of the route and the throughput without affecting the end-to-end delay. The parameters for simulation in NS-2.34 are presented in Table 2 . Since the value of density threshold $\lambda$ is basically dynamically calculated by each vehicle and set as threshold for the selection of next best fit forwarder, the same helps to know about the status of forward density connected.

Table 2. Simulation Parameters

\begin{tabular}{|c|c|}
\hline Simulation Parameters & Value \\
\hline Simulator & Ns-2(v2.34) \\
\hline SimulationTime & $500 \mathrm{~s}$ \\
\hline Arearange & $2000 \times 2000 \mathrm{~m}$ \\
\hline MaximumVelocity & $5-25 \mathrm{~m} / \mathrm{s}$ \\
\hline TransmissionRange & $200 \mathrm{~m}$ \\
\hline NumberOfVehicles & $15-100$ \\
\hline Numberoflane & 4 \\
\hline Bandwidth & $2 \mathrm{Mbps}$ \\
\hline InterfaceQueueLength & 50 \\
\hline Traffic Type & CBR \\
\hline PacketSize & $512 \mathrm{Bytes}$ \\
\hline PacketRate & $5 \mathrm{packets} / \mathrm{sec}$ \\
\hline PauseTime & $0 \mathrm{~s}$ \\
\hline BeaconInterval & $200 \mathrm{~ms}$ \\
\hline$\delta$ & $1 \mathrm{~m} / \mathrm{s}$ \\
\hline
\end{tabular}

Evaluation Metrics: The performance evaluation metrics are as follows

- Packet delivery ratio:- It is the ratio between total successfully received packets by the destination and total packets transmitted by the source.

- Packet lost ratio:- It is the ratio between total packet lost and the total packet sent.

- End to End Delay:- It is the average time taken by the packet to travel from source to the destination

In this paper we are considering three different scenarios for city environment as well as highway too. These scenarios are different to each other based on the vehicle density i.e. low, medium and high. Low density consists of 15 vehicle $/ \mathrm{km}$, medium consist of 45 vehicles $/ \mathrm{km}$ and high density consist of more than 60 vehicles $/ \mathrm{km}$. The source and 
destination vehicle remains in the simulation area for the simulation duration. The source vehicle stops the generation of the packets 30 second prior the simulation ends. The city scenario consists of 10 junctions. If the vehicle enters into the recovery mode and does not find the vehicle in its communication toward to the destination, than the packet hold duration $\mathrm{T}_{\mathrm{w}}$, in the simulation we consider 2 second.

Packet delivery ratio:-Figure 1 and 2 shows packet delivery ratio for three different scenarios of city and highway respectively by considering different densities of vehicles i.e. low, medium and high. For all the traffic density GPSR performance is very poor in term of packet delivery ratio for all three scenarios of city and highway with different density. The packet delivery ratio varies from 10-12\% for GPSR. Other side the performance variation of E-GyTAR in terms of packet delivery ratio for all three scenarios of city and highways with different density range varies from 48-82\%., while the packet delivery ratio varies from 51-90\% for PBDCR. We can see hear that the packet delivery ratio for PBDCR is better than both the approaches, just because of selection of next forwarder that try to select the next forwarder based on homogeneous property of the surrounding environment. The properties like density of the vehicle and the standard deviation of the average relative velocity with respect to the surrounding helps to maintain the connected path duration. On the other side GPSR approaches follow the greedy approach for the selection off the next forwarder, which may not guarantee of connected path duration. While E-GyTAR try to maintain the connected path from source to destination by following the improved greedy approach with the help of location services. As the density of the vehicle is going to increase the performance of the PBDCR is much better than the E-GyTAR and GPSR in terms of the packet delivery Ratio for both the scenarios as highways and city.

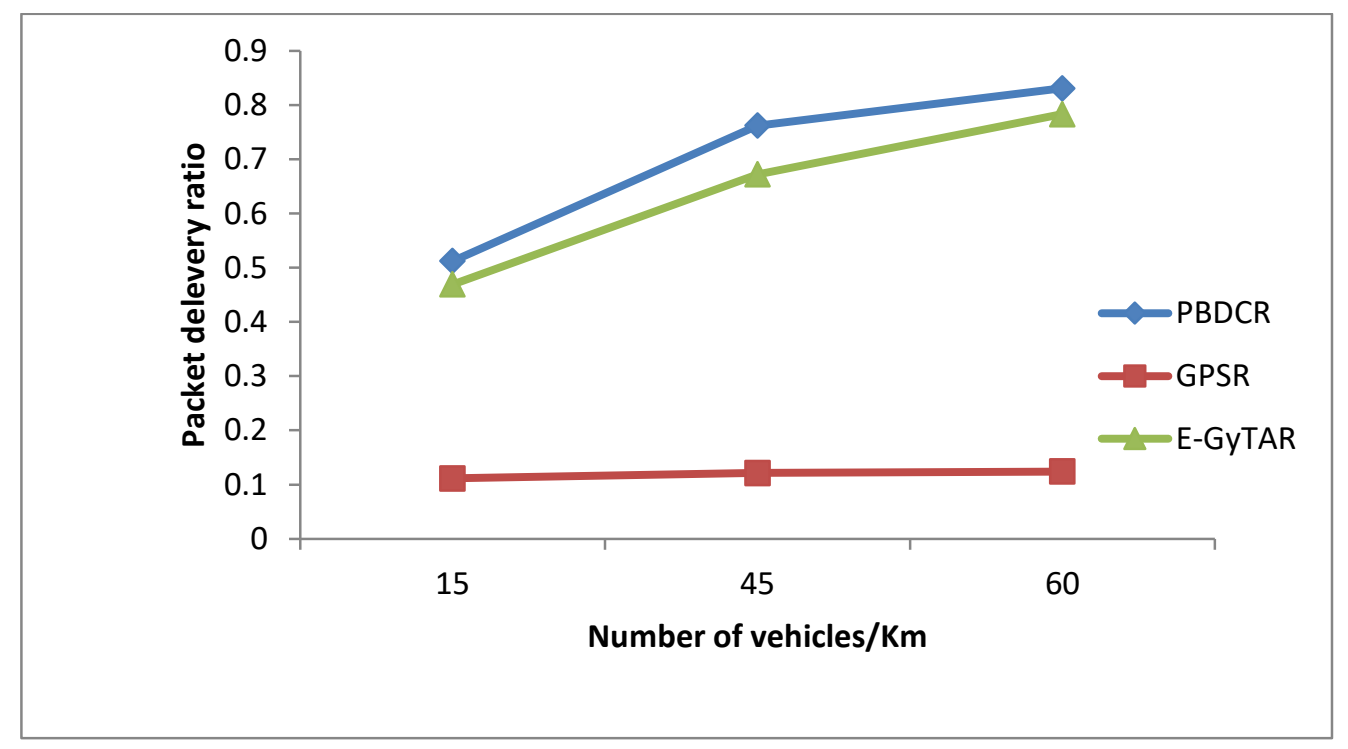

Figure 1. Number of Vehicles v/s Packet Delivery Ratio for City Scenarios 


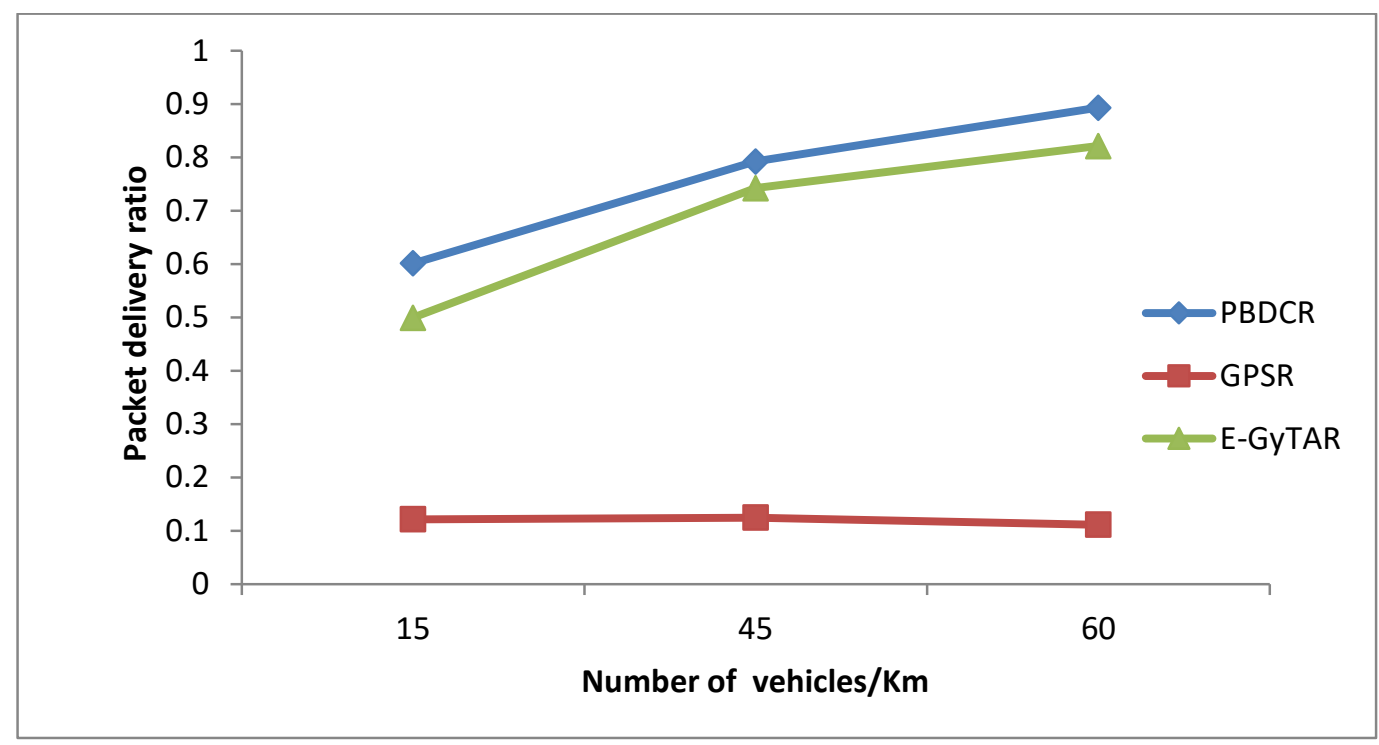

Figure 2. Number of Vehicles v/s Packet Delivery Ratio for Highway Scenarios

Average delay:- Figure 3 and 4 shows average data packet delay for three different scenarios of city and highway respectively by considering different densities of vehicles i.e. low, medium and high. For all the traffic density GPSR performance is very poor in term of average data packet delay for all three scenarios of city and highways with different density. The average data packet delay varies from 15-17 second for GPSR. Other side the performance variation of E-GyTAR in terms of average packet delay for all three scenarios of city and highways with different density range varies from 3.5-7 second. While the average packet delay varies from 2.5-6.5 second for PBDCR. Performance of PBDCR in Average data packet delay is better than the E-GyTAR and GPSR.

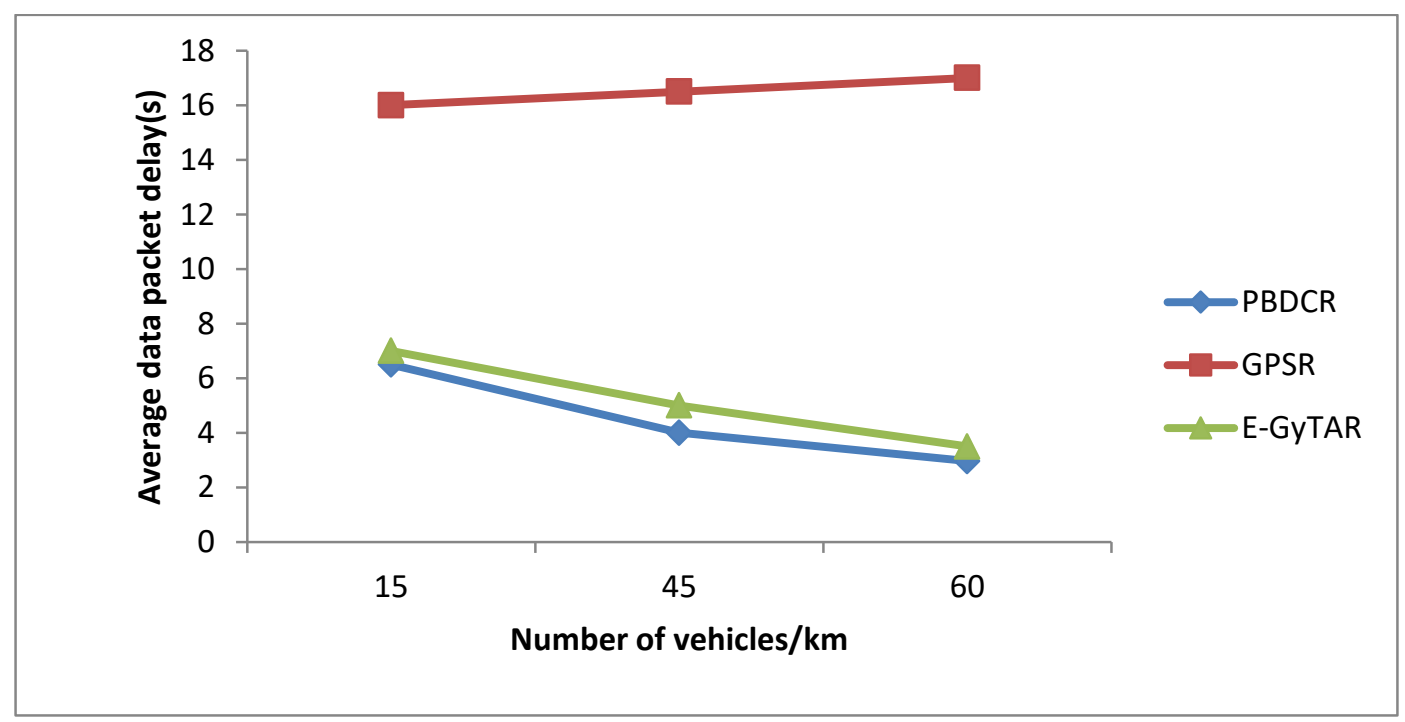

Figure 3. Number of Vehicles v/s Average Delay for City Scenarios 


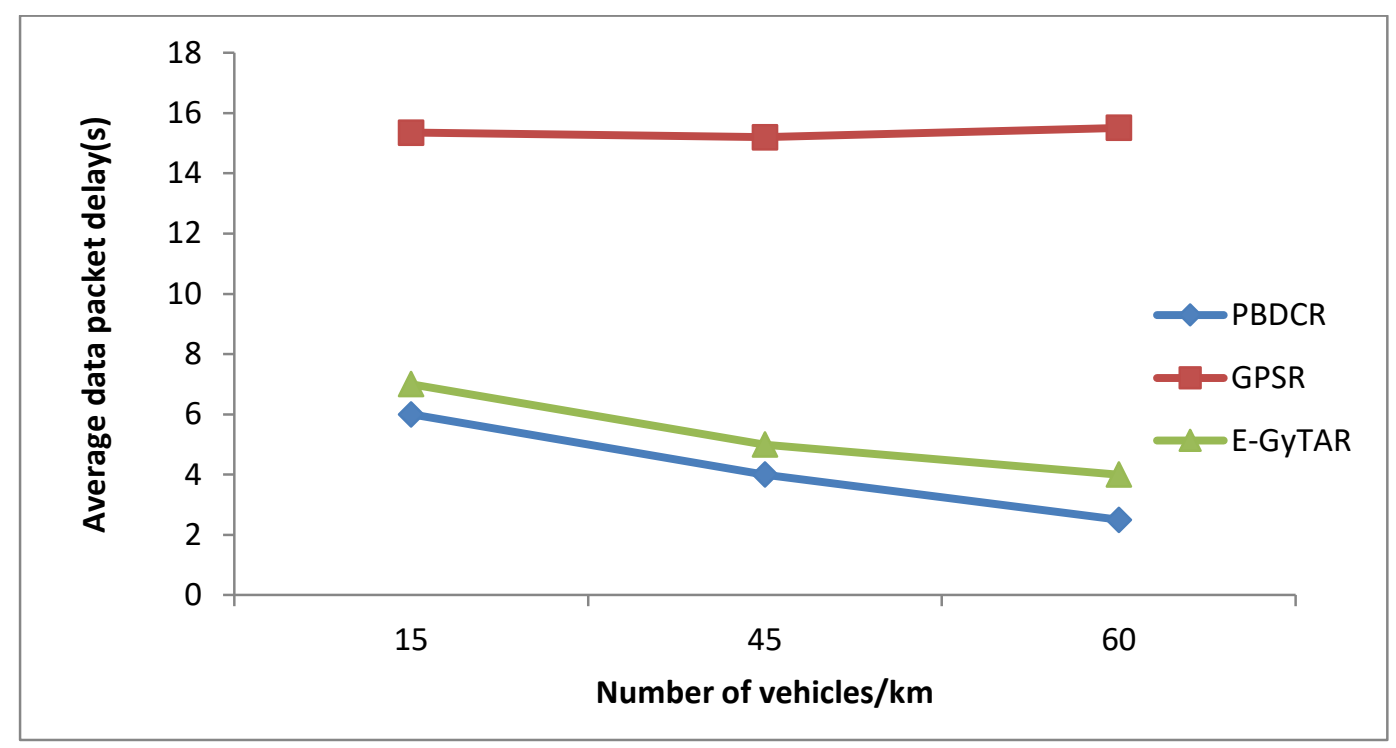

Figure 4. Number of Vehicles v/s Average Delay for Highway Scenarios

Packet lost ratio:- Figure 5 and 6 shows packet lost ratio for three different scenarios of city and highway respectively by considering different densities of vehicles i.e., low, medium and high. For all the traffic density GPSR performance is very poor in term of packet lost ratio for all three scenarios of city and highways with different density. The packet lost ratio varies from $88-90 \%$ for GPSR i.e., very high in term of data delivery from source and destination. Other side the performance variation of E-GyTAR in terms of average packet lost ratio for all three scenarios of city and highways with different density range varies from $18-52 \%$. While the average packet lost ratio varies from 10$50 \%$ for PBDCR. The performance of PBDCR is better than E-GyTAR and GPSR.

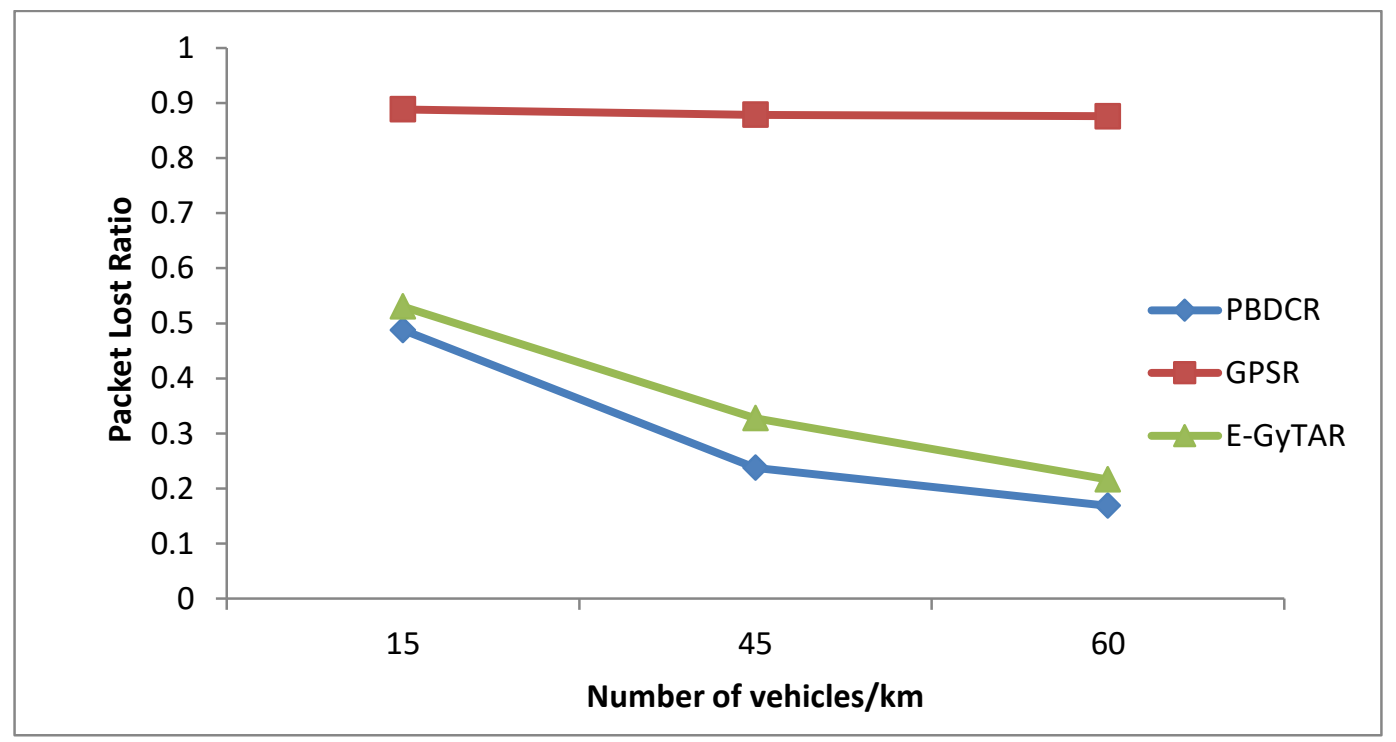

Figure 5. Number of Vehicles v/s Packet Lost Ratio for City Scenarios 


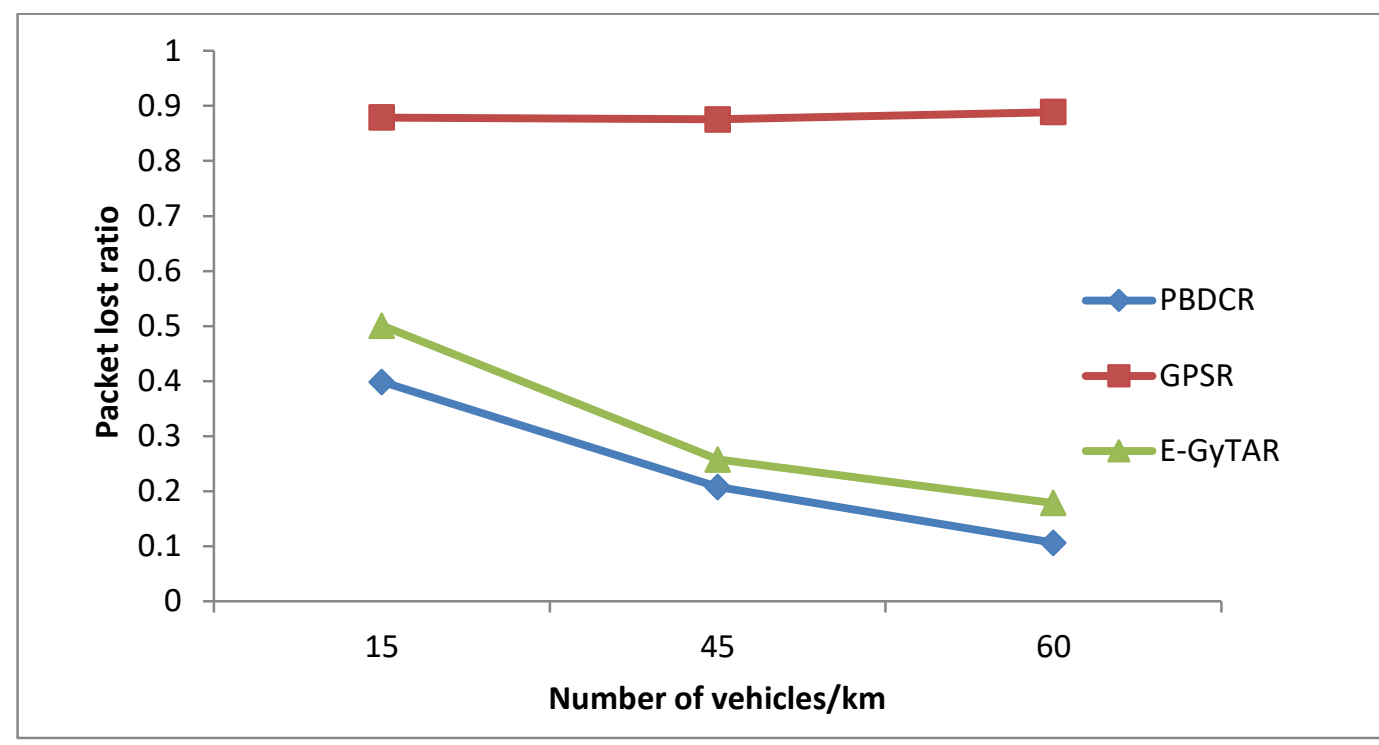

Figure 6. Number of Vehicles v/s Packet Lost Ratio for Highway Scenarios.

\section{Conclusion}

In this work, we proposed a position Based Density Conscious Routing Protocol which observes the neighborhood in the direction of the destination and selects the forwarder based on density and mobility. Thus, provides a better route life time with respect to EGyTAR and GPSR. We also examined the performance PBDCR with the E-GyTAR and GPSR routing protocol by simulation which reflects that, the proposed approach outperforms E-GyTAR and GPSR. The proposed work has been envisioned on a flat network and the extension of it on a hierarchical network e.g. clusters generated on density and mobility will be an interesting future work to explore.

\section{Appendix}

\section{Analytical Analysis}

Link Duration: The time duration for which the two forwarding vehicles in successive hops are connected to each other and are able to transfer the messages.

The messages are considered to have the following attributes

- Sender Identity::- The identity of the vehicle that generated the message i.e. the address of the source vehicle (Source ID).

- Receiver Identity::- The vehicle which is supposed to receive the message i.e. the address along with the position of the destination vehicle (Destination ID, Destination Position).

- Time to Live::- The time for which the packet will be considered as alive. It is identical to the traditional TTL parameter of Internet protocol packets.

This paper focuses on density based route discovery and maintenance for position based routing protocol for $\mathrm{V} 2 \mathrm{~V}$ communication. The vehicle updates its own position information by using GPS. Each vehicle periodically transmits its position and velocity information to its neighbors using beacon message. 


\section{Lemma 8: Link Life Time between Two Vehicles A and B}

Link Life Time (LLT) is the time for which the link between any two vehicles remains stable, where one vehicle is having the packet and then it selects the other one as the next best fit forwarder by using the above mentioned density based definitions. Two vehicles, say $A$ and $B$, which are in successive hops to forward a message from source to destination i.e., both, are contributing to form a path. Then, their link life time LLT $_{\mathrm{AB}}$

$L L T_{A B}=\frac{R-D_{A B}}{\left|v_{A}-v_{B}\right|}$

Where, $\mathrm{D}_{\mathrm{AB}}$ is the distance difference between vehicles $\mathrm{A}$ and $\mathrm{B}$ at any instant of time.

\section{Lemma 9: Route Life Time}

Route life time (RLT) for a route between source and destination will the minimum of LLTs between the successive hop vehicles forming the path. Suppose that the following vehicles form a path between source vehicle $S$ and destination vehicle $D$

$\mathrm{S} \rightarrow \mathrm{A} \rightarrow \mathrm{B} \rightarrow \mathrm{C} \rightarrow \mathrm{D}$

Then the route life time will be as follows:

$\operatorname{RLT}_{\mathrm{SD}}=\operatorname{Min}\left(\mathrm{LLT}_{\mathrm{SA}} ; \operatorname{LLT}_{\mathrm{AB}} ; \operatorname{LLT}_{\mathrm{BC}} ; \operatorname{LLT}_{\mathrm{CD}}\right)$

If two successive forwarders are having velocity difference very less than they will be in contact with each other for a longer duration. The standard deviation of average relative velocity of a vehicle signifies about the association of a vehicle with its surrounding. In case of sufficient density and less velocity variance indicates about to reduce the probability of the occurrence of the void region.

\section{Lemma 10: Link Reliability between Two Vehicles A and B}

Let at time $t_{1}$ the vehicle $A$ is ready to transmit the packet to destination and hence forward it to the next BFF vehicle say B. Suppose $\left(\mathrm{x}_{\mathrm{A}, \mathrm{t} 1}, \mathrm{y}_{\mathrm{A}, \mathrm{t} 1}\right)$ and $\left(\mathrm{x}_{\mathrm{B}, \mathrm{t} 1}, \mathrm{y}_{\mathrm{B}, \mathrm{t}}\right)$ be the initial positions of vehicles $\mathrm{A}$ and $\mathrm{B}$ respectively at time $\mathrm{t}_{1}$. In addition to it suppose that $v_{A}$ $\left(v_{A, x}, v_{A, y}\right)$ and $v_{B}\left(v_{B, x}, v_{B, y}\right)$ are the velocity vectors of vehicles $\mathrm{A}$ and $\mathrm{B}$ respectively. $\theta_{A B}$ is the angular displacement between two vehicles $\mathrm{A}$ and $\mathrm{B}$ during their movement in the same direction. Based on the Pythagorean Theorem, the physical distance between the vehicles can be expresses as the sum of the square of the minimum distance achieved and the distance of the mobile vehicles from the origin of a specific axis. At time $\mathrm{t}_{2}$ the distance difference between the vehicle $\mathrm{A}$ and $\mathrm{B}$ will be as follows

$$
d\left(t_{2}-t_{1}\right)=\sqrt{\begin{array}{c}
\left(\left(x_{B, t 1}-x_{A, t 1}\right)+\left(v_{B, x}-v_{A, x}\right)(t 2-t 1)\right)^{2} \\
+ \\
\left(\left(y_{B, t 1}-y_{A, t 1}\right)+\left(v_{B, y}-v_{A, y}\right)(t 2-t 1)\right)^{2}
\end{array}}
$$

Relative velocity between two vehicle A and B can be calculated as follows if the velocities are $\boldsymbol{v}_{\boldsymbol{A}}$ and $\boldsymbol{v}_{\boldsymbol{B}}$., respectively

$$
v_{A B}=\sqrt{v_{A}^{2}+v_{B}^{2}-2 v_{A} v_{B} \cos \theta_{A B}}
$$

The link reliability between two vehicles $\mathrm{A}$ and $\mathrm{B}$ can be expressed as the probability that they are under communication range of each other for the duration $T_{A B}=t_{2}-t_{1}$ where $\mathrm{T}_{\mathrm{AB}}$ is as follows: 
$T_{A B}=\frac{R-(d(t 2-t 1))}{\sqrt{v_{A}^{2}+v_{B}^{2}-2 v_{A} v_{B} \cos \theta_{A B}}}$

Link reliability between two vehicles $A$ and $B$ can be represented as follows:

$\mathrm{LR}\left(\mathrm{L}_{\mathrm{AB}}\right)=$ Prob $\left\{\right.$ Vehicles are connected at time $\mathrm{t}_{1}$ and they should be connected at the $\left.\mathrm{t}_{1}+\mathrm{T}_{\mathrm{AB}}\right\}$.

Since link reliability between two vehicles depends on their relative velocity. It is assumed that the velocity of vehicles has a normal distribution [14], therefore, the probability density function of velocity for the vehicle A will be as follows:

$f\left(v_{A}\right)=\frac{1}{\sigma_{A} \sqrt{2 \pi}} e^{-\left(\frac{v_{A}-\mu_{A}}{2 \sigma_{A}{ }^{2}}\right)^{2}}$

Probability density function of velocity for the vehicle B

$f\left(v_{B}\right)=\frac{1}{\sigma_{B} \sqrt{2 \pi}} e^{-\left(\frac{v_{B}-\mu_{B}}{2 \sigma_{B}{ }^{2}}\right)^{2}}$

Where $\mu_{A}$ and $\mu_{B}$ are the mean velocity of vehicles $\mathrm{A}$ and $\mathrm{B}$ respectively. Since velocity of individual vehicle in normally distributed then the relative velocity between two vehicles will be normally distributed. So based on the probability density function we can find the expected relative velocity between two vehicles A and B.

$E\left(v_{A B}\right)=\int_{-\infty}^{\infty} v_{A B} f\left(v_{A B}\right) d v_{A B}$

But probability density function for relative velocity between two vehicles A and B can be defined as follows

$f\left(v_{A B}\right)=f\left(v_{A}\right) f\left(v_{B}\right) f\left(\theta_{A B}\right)$

Since we are considering here highway as scenarios where vehicles are moving in the same direction. So the value of $\theta_{A B}$ can vary in the interval of $[0, \pi / 2]$. If we consider that $\theta_{A B}=0$ when two vehicles are moving in the same direction. For highway as scenarios the angle variation between two velocity vectors should be less than $18^{0}$.

Then two cases arises:

Case1:- If $v_{A}=v_{B}$ then

$v_{A B}=\sqrt{v_{A}^{2}+v_{B}^{2}-2 v_{A} v_{B} \cos \theta_{A B}}$ Will be equal to zero.

Therefore the expected relative velocity $E\left(v_{A B}\right)$ will be zero.

Case2:- If $v_{A} \neq v_{B}$ then

$v_{A B}=\sqrt{v_{A}^{2}+v_{B}^{2}-2 v_{A} v_{B} \cos \theta_{A B}}$ Since the velocity is normally distributed then the standard deviation of velocity can vary in the interval of [1 3 ] then let us assume that $v_{A}$ $=\alpha v_{B}$ and based on the above assumption

$v_{A B}=(\alpha-1) v_{B}$

Based on the above notion the expected relative velocity between two vehicles can be calculated as follows:

$$
\begin{gathered}
E\left(v_{A B}\right)=\int_{v_{A \min }}^{v_{A \max }} \int_{v_{B \min }}^{v_{B \max }}(\alpha-1) v_{B} \frac{1}{\sigma_{A} \sqrt{2 \pi}} e^{-\left(\frac{v_{A}-\mu_{A}}{2 \sigma_{A}{ }^{2}}\right)^{2}} \\
\frac{1}{\sigma_{B} \sqrt{2 \pi}} e^{-\left(\frac{v_{B}-\mu_{B}}{2 \sigma_{B}{ }^{2}}\right)^{2}} d v_{B} d v_{A}
\end{gathered}
$$

For every value of $\alpha>1$. 
When the relative distance between two vehicles changes from -R to $R$, let $f\left(T_{A B}\right)$ denote the probability density function of the communication duration then we can calculate $\mathrm{f}\left(\mathrm{T}_{\mathrm{AB}}\right)$ as follows:

$f\left(T_{A B}\right)=\frac{4 R}{\sigma_{v_{A B}} \sqrt{2 \pi}} \frac{1}{T_{A B}{ }^{2}} e^{-\left(\frac{\frac{2 R}{T_{A B}} E\left(v_{A B}\right)}{2 \sigma_{v}}{ }^{2}\right)^{2}}$ for $T_{A B}>0$

Where $\sigma_{v_{A B}}$ and $E\left(v_{A B}\right)$ are the variance and expected value of the relative velocity. Now we can integrate equ.(20) from $t_{1}$ to $t_{1}+T_{A B}$ to get the probability that at time $t_{1}$, the link will be available for the duration $\mathrm{T}_{\mathrm{AB}}$. Therefore the link reliability at time $\mathrm{t}_{1}$ will be as follows.

$r_{t 1}\left(l_{A B}\right)=\left\{\int_{t 1}^{t 1+T A B} f\left(T_{A B}\right) d T_{A B}\right.$ if $T_{A B}>0$ otherwise 0

Where $r\left(l_{A B}\right)$ is the link reliability between two vehicles A and B.

The above integration in equ.(21) can be derived using Gauss error function [14]. It can be obtained as follows

$$
\text { WhenT }_{A B} \geq 0
$$

$$
r_{t 1}\left(l_{A B}\right)=\operatorname{Erf}\left[\frac{\left[\frac{2 R}{t_{1}}-E\left(v_{A B}\right)\right.}{\sigma_{v_{A B}} \sqrt{2}}\right]-\operatorname{Erf}\left[\frac{\frac{2 R}{t_{1}+T_{A B}}-E\left(v_{A B}\right)}{\sigma_{v_{A B}} \sqrt{2}}\right]
$$

Based on the above mathematical formulation the Link Life Time between two vehicles $\mathrm{A}$ and $\mathrm{B}$ will be equal to the link reliability between both the vehicles i.e.,

$$
\operatorname{LLT}_{\mathrm{AB}}=r\left(l_{A B}\right)
$$

\section{References}

[1] T. Abdelkader, K. Naik, A. Nayak, N. Goel and V. Srivastava, "SGBR: A Routing Protocol for Delay Tolerant Networks Using Social Grouping”, IEEE Trans. Parallel and Distributed Systems, vol. 24, no. 12, (2013), pp. 2472-2481.

[2] B. C. Seet, G. Liu, B. S. Lee, C. H. Foh, K. J. Wong and K. K. Lee, "A-STAR: A Mobile ad hoc Routing Strategy for Metropolis Vehicular Communications”, Proc. Networking, (2004).

[3] M.-C. Chuang and M. C. Chen, "DEEP: Density Aware Emergency Message Extension Protocol for VANETs", IEEE Transaction on Wireless Communication, vol. 12, no. 10, (2013), pp. 4983-4993.

[4] Q. Fu, B. Krishnamachari and L. Zhang, "DAWN: A density adaptive routing for deadline-based data collection in vehicular delay tolerant networks", IEEE, Tsinghua Science and Technology, vol. 18, no. 3, (2013), pp. 230-241.

[5] M. Jerbi, S. M. Senouci, R. Meraihi and Y. Ghamri-Doudane, "An Improved Vehicular Ad Hoc Routing Protocol for City Environments”, IEEE International Conference on Communications, (2007).

[6] D. B. Johnson and D. A. Maltz, "Dynamic Source Routing in Ad Hoc Wireless Networks", Edited by Tomasz Imielinski and Hank Korth, Kluwer Acadmic Publishers, (1996), pp. 153-181.

[7] B. Karp and H. T. Kung, "GPSR: Greedy Perimeter Stateless Routing for Wireless Networks", Proceedings of the 6th Annual International Conference on Mobile Computing and Networking, MobiCom., (2000).

[8] J. B. Kenney, "Dedicated Short-Range Communications (DSRC) Standards in the United States", Proceedings of the IEEE, (2011).

[9] M. J. Khabbaz, H. M. Alazemi and C. M. Assi, "Delay-Aware Data Delivery in Vehicular Intermittently Connected Networks", IEEE Transactions on Communications, vol. 61, no. 3, (2013), pp. 1134-1143.

[10] M. J. Khabbaz, W. F. Fawaz and C. M. Assi, "Probabilistic Bundle Relaying Schemes in Two-Hop Vehicular Delay Tolerant Networks", IEEE Communications Letters, vol. 15, no. 3, (2011), pp. 281283.

[11] C. Lochert, H. Hartenstein, J. Tian, H. Fussler and D. Hermann, "A routing strategy for vehicular ad hoc networks in city environments", Proceedings. IEEE Intelligent Vehicles Symposium, (2003).

[12] C. Lochert, H. Hartenstein, J. Tian, H. Fubler and M. Mauve, "Geographic Routing in City Scenarios", ACM SIGMOBILE Mob. Comput. Commun. Rev., vol. 9, no. 1, (2005), pp. 69-72.

[13] V. Naumov and T. R. Gross, "Connectivity-Aware Routing (CAR) in Vehicular Ad-hoc Networks", IEEE INFOCOM, 26th IEEE International Conference on Computer Communications, (2007).

[14] Z. Niu, W. Yao, Q. Niand and Y. Song, "Link Reliability Model for Vehicle ad hoc Networks", London Communications Symposium, (2006) 
[15] V. D. Park and M. S. Corson, "A Performance Comparison of the Temporally-ordered Routing Algorithm and Ideal Link-state Routing", Proceedings Third IEEE Symposium on Computers and Communications, (1998).

[16] C. E. Perkins and E. M. Royer, "Ad-hoc On-demand Distance Vector Routing”, Proceedings WMCSA Second IEEE Workshop on Mobile Computing Systems and Applications, (1999).

[17] S. Yan, J. Xiao-Ye and C. Shan-Zhi, "AGP: An Anchor-geography based Routing Protocol with Mobility Prediction for VANET in City Scenarios", The Journal of China university of Posts Telecommuns., vol. 18, no. 1, (2011), pp. 112-117.

[18] Q. Yang, A. Lim., S. Li, J. Fang and P. agrawal, "ACAR: Adaptive Connectivity Aware Routing for Vehicular ad hoc Network in City Scenarios", Mobile Networks and Appllications, vol. 15, no. 1, (2010), pp. 36-59.

[19] S. Bilal, S. Madani and I. Khan, "Enhanced Junction Selection Mechanism for Routing Protocol in VANETs", The International Arab Journal of Information Technology, vol. 8, no. 4, (2011), pp. 422429.

\section{Authors}

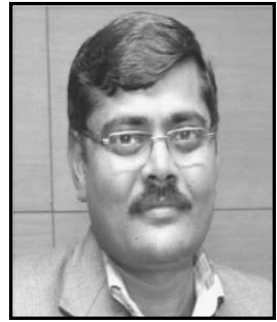

Anant Ram received Bachelor of Engineering in Computer Scienceand Engineering from Dr. BhimraoAmbedkar University, Agra, India in 2002 and Masterof Technology in Computer Science and Engineering from Uttar Pradesh TechnicalUniversity, Lucknow, India in the year 2008. Currently, he is a full time faculty and parttime Ph.D. Scholar at GLA University, Mathura, UP, India. His research interests areVANET, Distributed Systems, Mobile Computing, MANET and Data Mining.

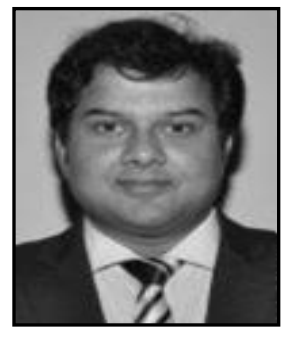

Manas Kumar Mishra received Bachelor of Engineering in Mechanical Engineeringfrom Shivaji University, Kolhapur, India in 1998, Master of Technology in SoftwareEngineering and $\mathrm{PhD}$ in Engineering with Computer Science as major from MotilalNehru National Institute of Technology Allahabad, India in the year 2003 and 2012,respectively. Currently, he is a full time faculty at GLA University, Mathura, UP, India.His research interests are Distributed Systems, Mobile Computing, MANET and Wireless Sensor Network. 
International Journal of Future Generation Communication and Networking Vol.10, No.9 (2017) 